\title{
Fast outflows and star formation quenching in quasar host galaxies $\star, \star \star$
}

\author{
S. Carniani ${ }^{1,2,3,4}$, A. Marconi ${ }^{1,2}$, R. Maiolino ${ }^{3,4}$, B. Balmaverde ${ }^{1}$, M. Brusa ${ }^{5,6}$, M. Cano-Díaz ${ }^{7}$, C. Cicone ${ }^{8}$,

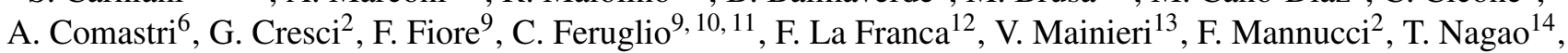 \\ H. Netzer ${ }^{15}$, E. Piconcelli ${ }^{9}$, G. Risaliti ${ }^{2}$, R. Schneider ${ }^{9}$, and O. Shemmer ${ }^{16}$
}

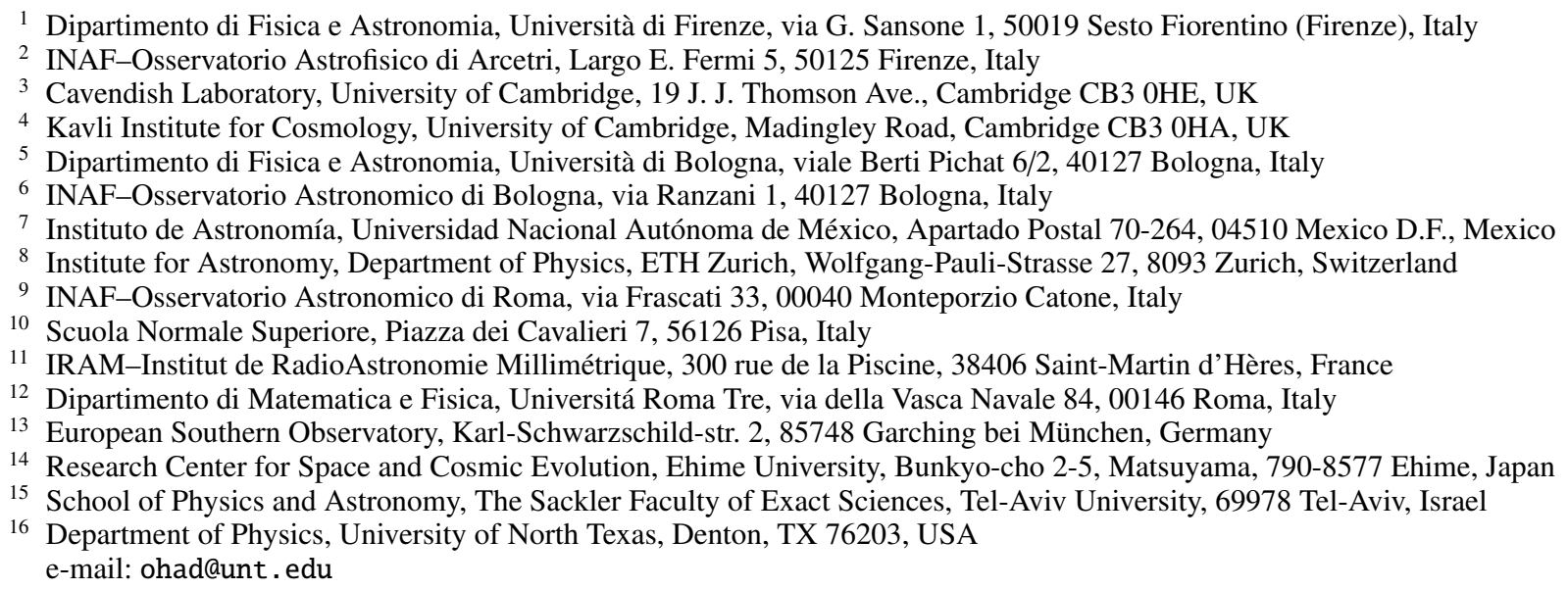

Received 23 December 2015 / Accepted 22 April 2016

\begin{abstract}
Negative feedback from active galactic nuclei (AGN) is considered a key mechanism in shaping galaxy evolution. Fast, extended outflows are frequently detected in the AGN host galaxies at all redshifts and luminosities, both in ionised and molecular gas. However, these outflows are only potentially able to quench star formation, and we are still lacking decisive evidence of negative feedback in action. Here we present observations obtained with the Spectrograph for INtegral Field Observations in the Near Infrared (SINFONI) $H$ and $K$-band integral-field of two quasars at $z \sim 2.4$ that are characterised by fast, extended outflows detected through the [OIII] $\lambda 5007$ line. The high signal-to-noise ratio of our observations allows us to identify faint narrow $\left(F W H M<500 \mathrm{~km} \mathrm{~s}^{-1}\right)$ and spatially extended components in [OIII] $\lambda 5007$ and $\mathrm{H} \alpha$ emission associated with star formation in the host galaxy. This star formation powered emission is spatially anti-correlated with the fast outflows. The ionised outflows therefore appear to be able to suppress star formation in the region where the outflow is expanding. However, the detection of narrow spatially extended $\mathrm{H} \alpha$ emission indicates star formation rates of at least $\sim 50-90 M_{\odot} \mathrm{yr}^{-1}$, suggesting either that AGN feedback does not affect the whole galaxy or that many feedback episodes are required before star formation is completely quenched. On the other hand, the narrow $\mathrm{H} \alpha$ emission extending along the edges of the outflow cone may also lead also to a positive feedback interpretation. Our results highlight the possible double role of galaxy-wide outflows in host galaxy evolution.
\end{abstract}

Key words. quasars: emission lines - galaxies: high-redshift - galaxies: active - galaxies: evolution

\section{Introduction}

A key problem in galaxy formation and evolution is understanding how active galactic nuclei (AGN) interact with their host galaxies. In particular, the origin of the tight correlations observed between the masses of supermassive black holes (BHs) and the stellar masses, stellar velocity dispersions, and luminosities of the host galaxy bulges is still debated

\footnotetext{
* Based on observations collected at the European Organisation for Astronomical Research in the Southern Hemisphere, Chile, P.ID: 086.B-0579(A) and 091.A-0261(A).

$\star \star$ The reduced data cubes are only available at the CDS via anonymous ftp to cdsarc.u-strasbg. fr (130.79.128.5) or via http://cdsarc.u-strasbg.fr/viz-bin/qcat?J/A+A/591/A28
}

(e.g. Magorrian et al. 1998; Ferrarese \& Merritt 2000; Gebhardt et al. 2000; Marconi \& Hunt 2003; Kormendy \& Ho 2013; Reines \& Volonteri 2015). Negative feedback from AGN can provide a viable explanation for these correlations (Fabian 2012; King \& Pounds 2015). According to theoretical models, AGNdriven outflows regulate $\mathrm{BH}$ growth and star formation activity in the host galaxies by blowing away the gas that feeds star formation and $\mathrm{BH}$ growth. Not only does negative feedback provide the link between the growth of supermassive black holes and their host galaxies, it also is a fundamental mechanism to explain the steep slope of the high end of the stellar mass function and to account for the existence of the red sequence of massive passive galaxies (e.g. Baldry et al. 2004; Hopkins et al. 2006; Pérez-González et al. 2008). 
Several theoretical models have been proposed for radiationpressure-driven outflows in AGN as possible feedback mechanism able to regulate $\mathrm{BH}$ and stellar mass accretion in active galaxies (e.g. Granato et al. 2004; Di Matteo et al. 2005; Menci et al. 2008; King 2010; Zubovas \& King 2012; Fabian 2012; Faucher-Giguère \& Quataert 2012; Zubovas \& King 2014; Nayakshin 2014; Costa et al. 2014, 2015; King \& Pounds 2015). Although AGN-driven outflows should be powerful enough to sweep away all gas from the host galaxy, recent simulations (e.g. Roos et al. 2015) and observations (e.g. Balmaverde et al. 2016) indicate that the effect of AGN feedback on the star formation in the host galaxy might be marginal. The effect of the feedback mechanism on the star formation history and host galaxy evolution is still an open question.

There is much evidence of AGN outflows of both molecular and ionised gas (e.g. Cicone et al. 2012, 2014, 2015; Maiolino et al. 2012; Cano-Díaz et al. 2012; Rupke \& Veilleux 2013; Feruglio et al. 2013b,a, 2015; Harrison et al. 2014, 2016; Aalto et al. 2015; Brusa et al. 2015; Perna et al. 2015a,b; Cresci et al. 2015a,b; Carniani et al. 2015). Molecular and ionised outflows are extended from a few hundred pc (e.g. Feruglio et al. 2015) to tens of kpc (e.g. Harrison et al. 2012; Cresci et al. 2015a) and are characterised by mass-loss rates that can exceed the star formation rate (SFR) by two orders of magnitude (Cicone et al. 2014). In particular, when molecular outflow rates are compared with molecular gas masses, gas depletion timescales (i.e. the time required for the outflow to completely expel the available gas) are much shorter than typical galaxy timescales, indicating that outflows are potentially able to provide the negative feedback required by the models (e.g. Cicone et al. 2014). This means that while these results suggest that molecular AGN-driven outflows can potentially quench star formation, we are still lacking direct evidence that they are effectively doing so.

As yet, there have only been a few tentative detections of the signature of AGN exerting a negative feedback on star formation in the host galaxy. In a luminous QSO at $z \sim 2.5$, CanoDíaz et al. (2012) presented evidence that $\mathrm{H} \alpha$ emission tracing star formation is suppressed in the region affected by fast outflows that is traced by [OIII] $\lambda 5007$ emission. A similar result was found by Cresci et al. (2015a) in an obscured QSO at $z \sim 1.5$, where the ionised outflow appears to be sweeping away the gas in the host galaxy and thus quenches star formation. Given the low signal-to-noise ratio $(\mathrm{S} / \mathrm{N})$ of the star-formationpowered $\mathrm{H} \alpha$ detected by Cano-Díaz et al. (2012) and by Cresci et al. (2015a), we need to confirm these findings with higher $\mathrm{S} / \mathrm{N}$ and in a larger sample of objects. Moreover, the $\mathrm{H} \alpha$ emission in the two host galaxies of these QSOs indicates remarkably high SFRs of $\sim 100 M_{\odot} \mathrm{yr}^{-1}$. Therefore, it is still unclear whether these AGN-driven outflows are able to completely quench SF in the host galaxy, as required by models.

In this work we present new observations made with the Spectrograph for INtegral Field Observations in the Near Infrared (SINFONI) $K$-band of two quasars at $z \sim 2.4$ : LBQS 0109+0213 (hereafter LBQS0109) and HB89 0329-385 (hereafter HB8903). These objects are part of a larger sample observed with SINFONI in the $H$-band with the aim of mapping the kinematics of the [OIII] $\lambda 5007$ line (Carniani et al. 2015, hereafter Paper I). Their spatially resolved [OIII] 25007 kinematics clearly reveals extended ionised outflows with velocities exceeding $1000 \mathrm{~km} \mathrm{~s}^{-1}$. In addition to the broad and bright outflowing component of [OIII] $\lambda 5007$, these objects are characterised by faint, narrow [OIII] $\lambda 5007$ lines that are most likely associated with star formation powered emission in the host galaxies. The aim of the Very Large Telescope (VLT)/SINFONI $K$-band observations is to detect the corresponding narrow $\mathrm{H} \alpha$ components to verify the star formation origin and study its spatial distribution compared to the outflow location, as was done in Cano-Díaz et al. (2012) and Cresci et al. (2015a).

The paper is organised as follows: in Sect. 2 we present the properties of the K SINFONI datasets, and in Sect. 3 we show the analysis of the $H$ - and $K$-band spectra aimed at revealing narrow [OIII] $\lambda 5007$ and $\mathrm{H} \alpha$ components. The spatial distribution of the two narrow components in both QSOs is discussed in Sect. 4. Finally, the conclusions are presented in Sect. 5. A $H_{0}=67.3 \mathrm{~km} \mathrm{~s}^{-1} \mathrm{Mpc}^{-1}, \Omega_{\mathrm{M}}=0.315, \Omega_{\Lambda}=0.685$ cosmology is adopted throughout this work (Planck Collaboration XVI 2014).

\section{Observations and data reduction}

The two sources LBQS0109 and HB8903 were observed with the SINFONI at the VLT. Observations from program 091.A0261(A) (PI A. Marconi) were executed in seeing-limited mode $\left(\leq 0^{\prime \prime} .6\right)$ with $00^{\prime \prime} 250$ spatial scale and the $K$-band grating, with a spectral resolution of $R=4000$ over the observed wavelength range $\lambda=1.95-2.45 \mathrm{~nm}$. The observations of each target were divided into six $1 \mathrm{~h}$ long observing blocks (OB) for a total onsource integration time of four hours (plus overheads). During each observing block, an ABBA nodding was adopted to perform sky subtraction. Standard stars for telluric correction and flux calibration were observed shortly after or before the execution of each OB. $K$-band data were reduced using the standard ESO-SINFONI pipeline 2.6.0 after removing cosmic-ray hits from the raw data with the L.A. Cosmic procedure by van Dokkum (2001). Sky subtraction was optimised using the IDL routine by Davies (2007). The final data cubes have a spatial scale of $0^{\prime \prime} .125 \times 00^{\prime \prime} .125$ and a field of view of $8^{\prime \prime} \times 8^{\prime \prime}$. However, all figures in this paper will only show a fraction of the field of view $\left(2^{\prime \prime} \times 2^{\prime \prime}\right)$, since observed emissions are extended for less than $\sim 1^{\prime \prime}(\sim 8 \mathrm{kpc})$. The estimated angular resolution is $\sim 0^{\prime \prime} 6$, based on a 2D Gaussian fitting of the flux map of the spatially unresolved broad $\mathrm{H} \alpha$ line (see Sect. 4 for a description of the fitting procedure).

In addition to the $K$-band dataset, we also considered the $H$-band SINFONI spectra from program 086.B-0579(A), which are described in detail in Paper I.

\section{Data analysis}

In Paper I we presented $H$-band SINFONI spectra of five QSOs at $z \sim 2.4$ that were used to map extended outflows traced by the [OIII] $\lambda 5007$ emission line. The broad blue wings of the [OIII] $\lambda 5007$ line indicated outflow velocities of 500-2300 $\mathrm{km} \mathrm{s}^{-1}$. By applying a new method, based on spectroastrometry of the [OIII] $\lambda 5007$ line, we found that the spatial centroids of the emission extracted from blue-shifted spectral channels are displaced with respect to the QSO position, suggesting that the outflowing gas is extended a few kpc from the centre and is not isotropically distributed (Fig. 4 of Paper I). We inferred ionised outflow rates of $\sim 10-700 M_{\odot} \mathrm{yr}^{-1}$. Both outflow velocities and mass rates increase with AGN bolometric luminosity, suggesting a correlation between the two quantities. In this work we present new observations of two QSOs, LBQS0109 and HB8903, belonging to the sample of Paper I, whose characteristics are summarised in Table 1. 
Table 1. Quasar properties.

\begin{tabular}{|c|c|c|c|c|c|c|c|c|c|}
\hline QSO & (2) & RA & Dec & $\begin{array}{c}\log _{10}\left(\frac{L_{\mathrm{AGN}}}{\mathrm{erg} / \mathrm{s}}\right) \\
(5)\end{array}$ & $\begin{array}{c}v_{\text {outflow }} \\
{\left[\mathrm{km} \mathrm{s}^{-1}\right]} \\
(6)\end{array}$ & $\begin{array}{c}R_{\text {outflow }} \\
{[\mathrm{kpc}]} \\
(7)\end{array}$ & $\begin{array}{c}M_{[\mathrm{OIII}]}^{\text {outflow }} \\
{\left[10^{7} M_{\odot}\right]} \\
(8)\end{array}$ & $\begin{array}{c}\dot{M}_{[\mathrm{OIII}]}^{\text {outflow }} \\
{\left[M_{\odot} \mathrm{yr}^{-1}\right]} \\
(9)\end{array}$ & $\begin{array}{c}M_{\mathrm{BH}} \\
{\left[10^{10} M_{\odot}\right]} \\
(10)\end{array}$ \\
\hline LBQS0109 & 2.35 & $01: 12: 16.9$ & $+02: 29: 47$ & 47.5 & 1850 & 0.4 & 1.2 & 60 & 1.0 \\
\hline HB8903 & 2.44 & 03:31:06.41 & $-38: 24: 04.6$ & 47.5 & 1450 & 1.9 & 0.7 & 6 & 1.3 \\
\hline
\end{tabular}

Notes. (1) ID of the object. (2) Red-shifts are estimated from [OIII] $\lambda 5007$. (3), (4) Coordinates. (5) AGN bolometric luminosities are derived using the relation $L_{\mathrm{AGN}} \sim 6 \lambda L(\lambda 5100 \AA$ ) from Marconi et al. (2004). (6), (7) Outflow velocities and radii are inferred with the spectroastrometric method described in Paper I. (8) Outflow masses are inferred from [OIII] $\lambda 5007$ assuming a $T_{\mathrm{e}} \sim 10^{4} \mathrm{~K}$ and a $n_{\mathrm{e}} \sim 500 \mathrm{~cm}^{-3}$. (9) Outflow mass rates are calculated as $\dot{M}^{\text {outflow }}=M^{\text {outflow }} v_{\text {outflow }} / R_{\text {outflow }}$. (10) Black hole masses are adopted from Shemmer et al. (2004).

\subsection{Narrow [OIII] $] 5007$ components}

In the two QSOs, we detect a weak, narrow $(F W H M<$ $500 \mathrm{~km} \mathrm{~s}^{-1}$ ) component of the [OIII] $\lambda 5007$ emission that is only visible in some parts of the field of view. The narrow components are on the red side of the [OIII] $\lambda 5007$ profile. In Fig. 1 we compare for both QSO host galaxies the spectrum extracted from a region of $\sim 4 \mathrm{kpc} \times 4 \mathrm{kpc}$ centred on the AGN position (red profiles) with the spectra extracted from external regions of the host galaxy (blue profiles). The regions from which we extracted the spectra are shown in the left panels of Fig. 1. The narrow [OIII] $\lambda 5007$ component (hereafter [OIII] $]_{\text {nar }}$ ), identified in the figure by a dashed green line, is clearly visible in the eastern $(\mathrm{E})$ side for LBQS0109 and in the western (W) side for HB8903, showing that in these regions the [OIII] $\lambda 5007$ profile is different from the one extracted from the nuclear region. We note that the blue-shifted narrow emission detected in the lower left boxes of HB8903 is mostly due to outflow emission since the velocity dispersion in this region $\left(F W H M \sim 800 \mathrm{~km} \mathrm{~s}^{-1}\right)$ is larger than that observed in the other external regions $\left(F W H M \sim 500 \mathrm{~km} \mathrm{~s}^{-1}\right)$; we refer to Paper I for more details. In Paper I we showed that a broken power-law parametric profile represents a good fit to the main [OIII] $\lambda 5007$ component and, in particular, that it is sufficient to reproduce the spectra of nuclear and outflow regions (e.g. Fig. 1 of Paper I). However, a more complex line profile should be used to describe the spectra extracted from external regions where $[\mathrm{OIII}]_{\text {nar }}$ is visible (Fig. 1). For this reason, we reanalysed the $H$-band spectra and performed a new pixel-bypixel fitting by adding an additional narrow, red-shifted component to the parametric profile used in Paper I. Since the $\mathrm{S} / \mathrm{N}$ of $[\mathrm{OIII}]_{n a r}$ in each spatial pixel is low $(S / N<9)$, we cannot perform a kinematical analysis of this new component. Therefore, its velocity and width were fixed to the values obtained from a Gaussian fit of the line profile obtained by subtracting the nuclear spectrum $\left(r<0.5^{\prime \prime}\right)$ from the circumnuclear spectrum $\left(0.5^{\prime \prime}<r<1^{\prime \prime}\right)$, after re-scaling to match the intensities of the broad $\mathrm{H} \beta$ and [OIII] $\lambda 5007$ lines (Fig. 2). The best-fit results are listed in Table 2 for both targets.

The flux maps of the $[\mathrm{OIII}]_{\text {nar }}$ components are shown in Fig. 4. The narrow emission is not symmetrically distributed around the QSO. This property was evident in Fig. 1: the peak emission of [OIII $]_{\text {nar }}$ for LBQS0109 is visible E of the AGN location (at 0,0 position that is identified with a red diamond) and that of HB8903 is N and W of the AGN. These narrow components are not detected in $\mathrm{H} \beta$ probably because the $\mathrm{S} / \mathrm{N}$ of the data is lower.

The kinematics of the broad [OIII] $\lambda 5007$ components obtained after subtracting [OIII $]_{\text {nar }}$ are shown in Fig. 3. They are similar to those shown in Paper I and in the left panels of Fig. 1, indicating that they are not significantly affected by the narrow components.

The comparison of the $[\mathrm{OIII}]_{\text {nar }}$ flux maps with the velocity maps of the broad [OIII] 25007 , whose negative velocity are shown in blue contours in Fig. 4, reveals that the emission of the two narrow components is not spatially correlated with the ionised outflows. The surface brightness of [OIII $]_{\text {nar }}$ seems to be attenuated in the S-W region of LBQS0109 and in the S-E region of HB8903: both regions are characterised by high blueshifted velocity associated with the ionised outflow approaching along the line of sight.

By applying the spectroastrometric method to the [OIII] $\lambda 5007$ emission line, we found in Paper I that the photocentre position extracted from the blue side of the line profile is displaced with respect to the QSO position (Fig. 4 of Paper I). This offset is the signature of outflowing gas moving away from the nuclear region. In several cases, including the two QSO presented here, we also observed an offset on the red side of the line profile, indicating that ionised gas at red-shifted velocities is also emitted on galactic scales, far from the QSO position. Since [OIII] $]_{\text {nar }}$ emission detected in these two targets peaks in the same region (N-E for LBQSO0109 and W for HB8903) as the position of the red photocentres, it is very likely that the $[\mathrm{OIII}]_{\text {nar }}$ is the origin of the displacement observed on the red side. A narrow component may then be present in the other sources of our sample, but it is too weak to be clearly deblended either from the stronger nuclear [OIII] $\lambda 5007$ component or from the broad [OIII] 55007 outflow component, which may contaminate the red side profile.

The $[\mathrm{OIII}]_{\text {nar }}$ spatial extension well over $4 \mathrm{kpc}$ excludes the possibility that this line is emitted by NLR, which usually extends from a few hundred pc to a few kpc. (see e.g. Netzer et al. 2004; Husemann et al. 2014; Young et al. 2014). Both the line widths $\left(F W H M \sim 480 \mathrm{~km} \mathrm{~s}^{-1}\right)$ and the line strengths of the $[\mathrm{OIII}]_{\text {nar }}$ compared to the main broad [OIII] $\lambda 5007$ component suggest that these narrow components might be associated with star-forming regions in the galactic disks.

\subsection{K-band spectral fitting and the narrow $\mathrm{H} \alpha$ component}

Strong $\mathrm{H} \alpha$ emission is detected in both sources. The spectra extracted from a nuclear region $\left(0.25^{\prime \prime} \times 0.25^{\prime \prime}\right)$ show a very broad $\mathrm{H} \alpha$ profile (Fig. 5). From comparing the $\mathrm{H} \alpha$ profile with the $[\mathrm{OIII}] \lambda 5007$ and $\mathrm{H} \beta$ profiles analysed in Paper I, we expect a broad $\left(F W H M>4000 \mathrm{~km} \mathrm{~s}^{-1}\right)$ component originating from the BLR and a narrower component $\left(500 \mathrm{~km} \mathrm{~s}^{-1}<\right.$ $F W H M<2000 \mathrm{~km} \mathrm{~s}^{-1}$ ) overlapping with the [OIII] $\lambda 5007$ outflow. To set up the spaxel-by-spaxel fit for both targets, we initially considered the spectrum extracted from the nuclear aperture. Continuum emission was fitted with a single power law, 

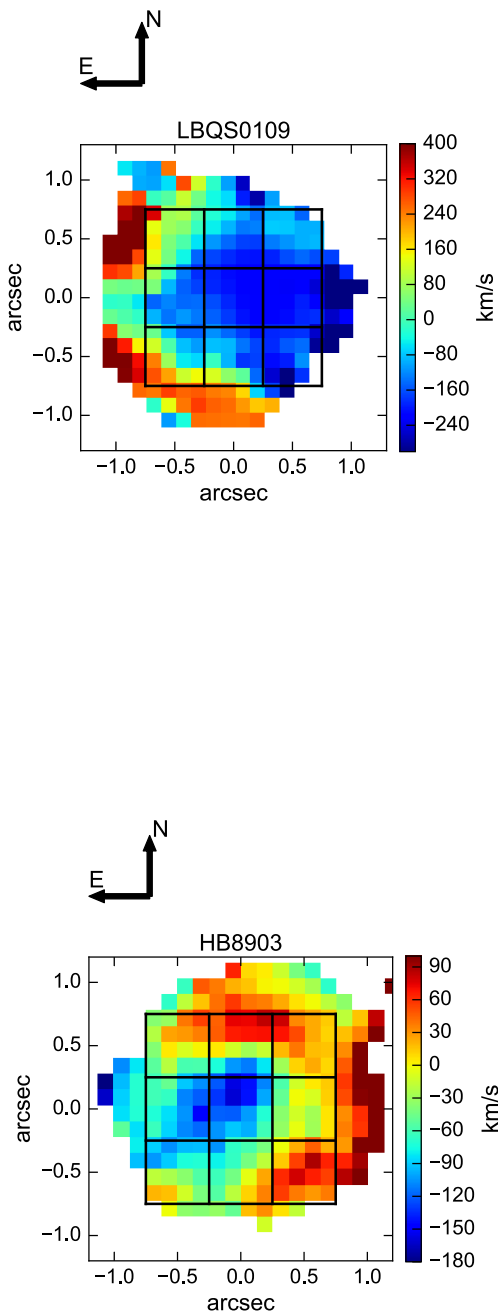

Fig. 1. Left panels: [OIII] $\lambda 5007$ velocity maps of LBQS0109 (top) and HB8903 (bottom) presented in Paper I. Black squares indicate the nine regions where we extracted the corresponding spectra shown in the right panels. Right panels: comparison between the nuclear [OIII] $\lambda 5007$ profiles (red) and those from the external regions (blue) of LBQS0109 (top) and HB8903 (bottom). The black dotted line corresponds to the [OIII] $\lambda 5007$ central wavelength. Narrow ( FWHM $\sim 490 \mathrm{~km} \mathrm{~s}^{-1}$ for LBQS0109 and $F W H M \sim 480 \mathrm{~km} \mathrm{~s}^{-1}$ for HB8903) components of the [OIII] $\lambda 5007$ emission, identified by the green dashed line, are clearly detected.

Table 2. Central wavelengths and line widths of the two narrow components of [OIII $]_{\text {nar }}$ and $\mathrm{H} \alpha_{\text {nar }}$.

\begin{tabular}{ccc|cc}
\hline \hline & {$[\mathrm{OIII}]_{\mathrm{nar}}$} & \multicolumn{2}{c}{$\mathrm{H} \alpha_{\text {nar }}$} \\
\hline & $\lambda_{0}$ & $F W H M$ & $\lambda_{0}$ & $F W H M$ \\
& {$[\mu \mathrm{m}]$} & {$\left[\mathrm{km} \mathrm{s}^{-1}\right]$} & {$[\mu \mathrm{m}]$} & {$\left[\mathrm{km} \mathrm{s}^{-1}\right]$} \\
\hline LBQS0109 & $1.6802 \pm 0.0004$ & $490 \pm 90$ & $2.203 \pm 0.001$ & $250 \pm 200$ \\
HB8903 & $1.7214 \pm 0.0002$ & $480 \pm 70$ & $2.2560 \pm 0.0006$ & $500 \pm 150$ \\
\hline
\end{tabular}

while the $\mathrm{H} \alpha$ line was fitted with broken power-law profiles for the BLR component and for the narrower NLR component associated with the outflow. In Fig. 5 the BLR and NLR components are labelled A and B, respectively. The [NII] doublet associated with component B was fitted with two Gaussian components (labelled C and D), and their velocities and widths were fixed to those of component B. Since the two [NII] emission lines $(\lambda \lambda 6584,6548 \AA)$ originate from the same upper level, their intensity ratio was fixed at the value of 3 , given by the ratio of the Einstein coefficients. For HB8903, the fitting procedure did not require the addition of the [NII] components to reproduce the $\mathrm{H} \alpha$ profile. In addition to the previously mentioned 

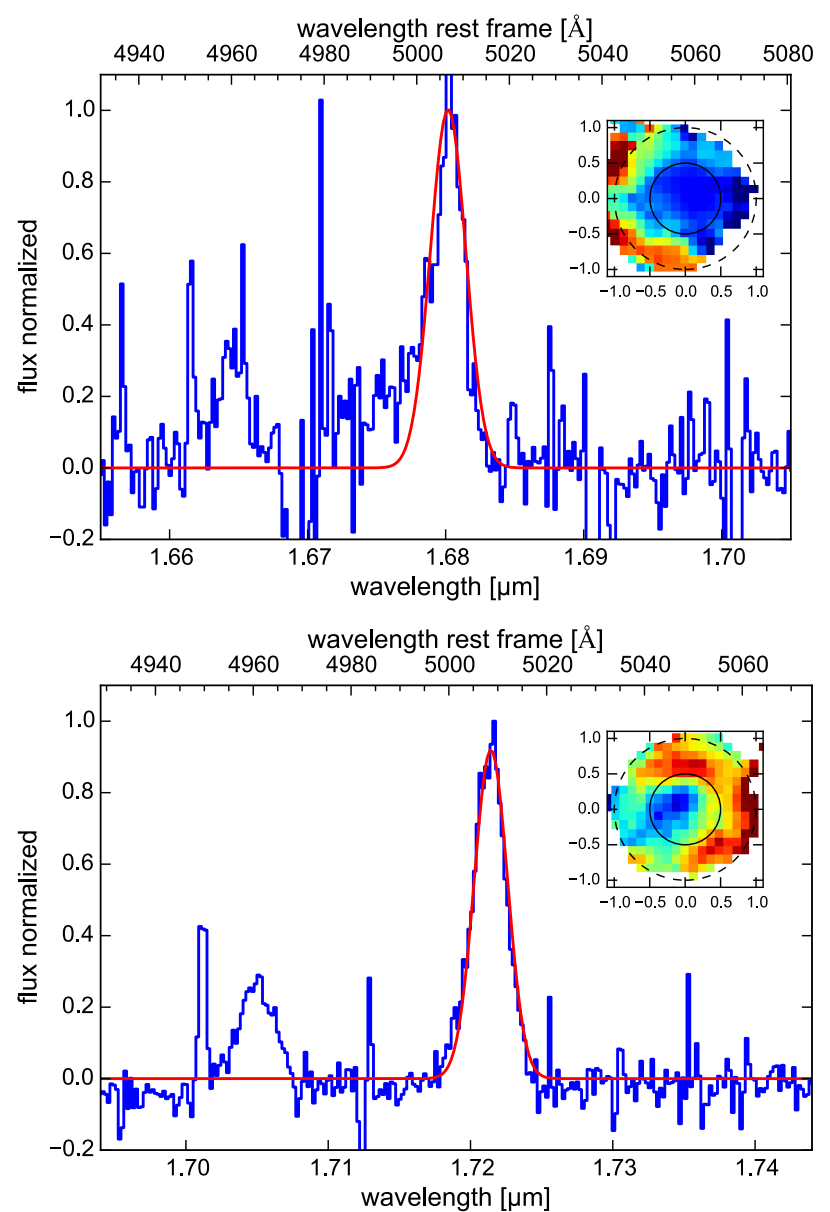

Fig. 2. $H$-band SINFONI spectra of LBQS0109 (top) and HB8903 (bottom). The observed spectra (blue lines) are the result of the subtraction between the spectra extracted from the ring-shaped regions $\left(0.5^{\prime \prime}<r<1^{\prime \prime}\right)$ and from the nuclear regions $\left(r<0.5^{\prime \prime}\right)$. The narrow doublet $[\mathrm{OIII}] \lambda 5007$ is evident in both sources. The red curves denote the Gaussian fits whose parameters are shown in Table 2. In the smaller inset, the nuclear region (solid black line) and the ring-shaped region (dashed black line) are drawn in the [OIII] $\lambda 5007$ velocity maps.

components (A, B, C, and D for LBQS0109 and A and B for HB8903), we added one Gaussian component for LBQS0109 (component $X$ in the left panel of Fig. 5) and two Gaussian components for HB8903 ( $X$ and $Y$ in the right panel of Fig. 5). The $Y$ component may be associated either with the broad HeI line at $\lambda=6679.99 \AA$ or the doublet [SII] $\lambda \lambda 6717,6731 \AA$. The wavelength of component $X$ in both sources cannot be associated with any known emission line, but it was added to reduce the $\chi^{2}$ of our fit. The fit residuals shown in Fig. 5 indicate that the adopted components describe the nuclear spectrum of each target well.

We performed the spectral fitting on individual spaxels by fixing the kinematics of the broad components and by allowing the flux (i.e. the amplitude) to vary. Figure 6 shows the residual spectra of the pixel-by-pixel fitting extracted from a ring-shaped region $\left(0.5^{\prime \prime}<r<0.8^{\prime \prime}\right)$ of the field of view. These residual spectra reveal a weak but significant narrow $\left(F W H M \sim 250 \mathrm{~km} \mathrm{~s}^{-1}\right.$ for LBQS0109 and $F W H M \sim 500 \mathrm{~km} \mathrm{~s}^{-1}$ for HB8903; see Table 2) $\mathrm{H} \alpha$ component. The velocity of this narrow component (hereafter $\mathrm{H} \alpha_{\text {nar }}$ ) is consistent within the errors with the red-shift of the narrow [OIII $]_{\text {nar }}$ detected in the $H$-band spectra (Sect. 3.1): the red arrow in Fig. 6 denotes the velocity of $[\mathrm{OIII}]_{\mathrm{nar}}$. The red dashed lines show the expected
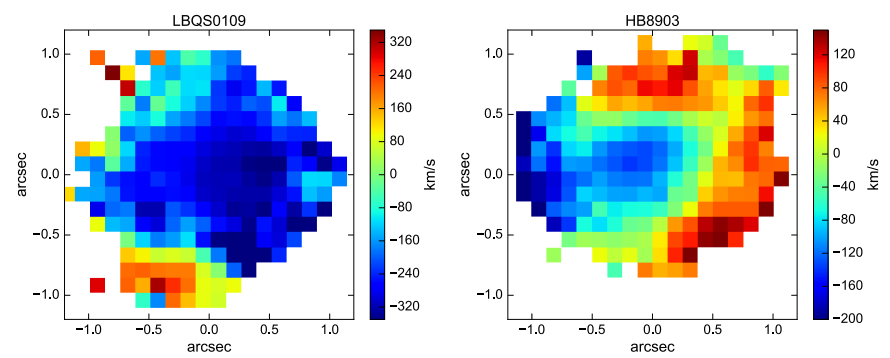

Fig. 3. [OIII] $] 5007$ median velocity maps of LBQS0109 (left) and HB8903 (right). The velocity maps are obtained considering only the broad [OIII] $\lambda 5007$ component, and they are therefore not influenced by the narrow $[\mathrm{OIII}] \lambda 5007$. They are to be compared with the median velocity maps obtained for the overall [OIII] $\lambda 5007$ profile shown in Fig. 1.
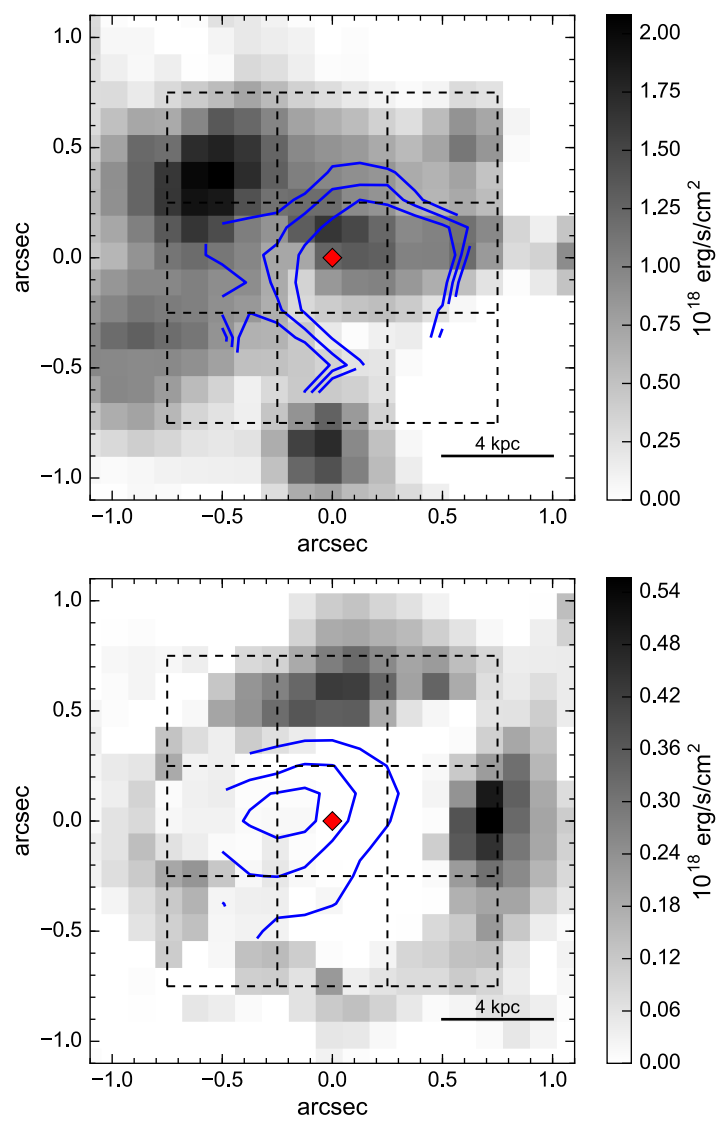

Fig. 4. Flux maps of the [OIII $]_{\text {nar }}$ component for LBQS0109 (top) and for HB8903 (bottom) obtained from the fit described in the text. The AGN position is identified with a red diamond. The blue contours trace the [OIII] $\lambda 5007$ blue-shifted emission cone shown in Fig. 3. The contours represent the velocity $-300,-275$ and $-250 \mathrm{~km} \mathrm{~s}^{-1}$ for LBQS0109, and $-125,-100$ and $-50 \mathrm{~km} \mathrm{~s}^{-1}$ for HB8903. The [OIII $]_{\text {nar }}$ emissions are not symmetrically distributed and are anti-correlated with the outflow region. Black squares indicate the nine regions where we extracted the corresponding spectra shown in the right panels of Fig. 1.

position of the corresponding narrow $[\mathrm{NII}]_{\text {nar }}$ components associated with $\mathrm{H} \alpha_{\text {nar }}$. The doublet $[\mathrm{NII}]_{\text {nar }}$ is clearly not detected in the residual spectra of either object, resulting in an upper limit of $\log _{10}\left([\mathrm{NII}]_{\mathrm{nar}} / \mathrm{H} \alpha_{\mathrm{nar}}\right)<-0.85$ for LBQS0109 and of $\log _{10}\left([\mathrm{NII}]_{\text {nar }} / \mathrm{H} \alpha_{\text {nar }}\right)<-1.32$ for HB8903. By studying the line ratios using the Baldwin, Phillips \& Terlevich (BPT) method (Baldwin et al. 1981), we find that the narrow emission is consistent with gas excited by the star formation region (Kauffmann et al. 2003). Moreover, the $F W H M \lesssim 500 \mathrm{~km} \mathrm{~s}^{-1}$ of the $\mathrm{H} \alpha$ nar 

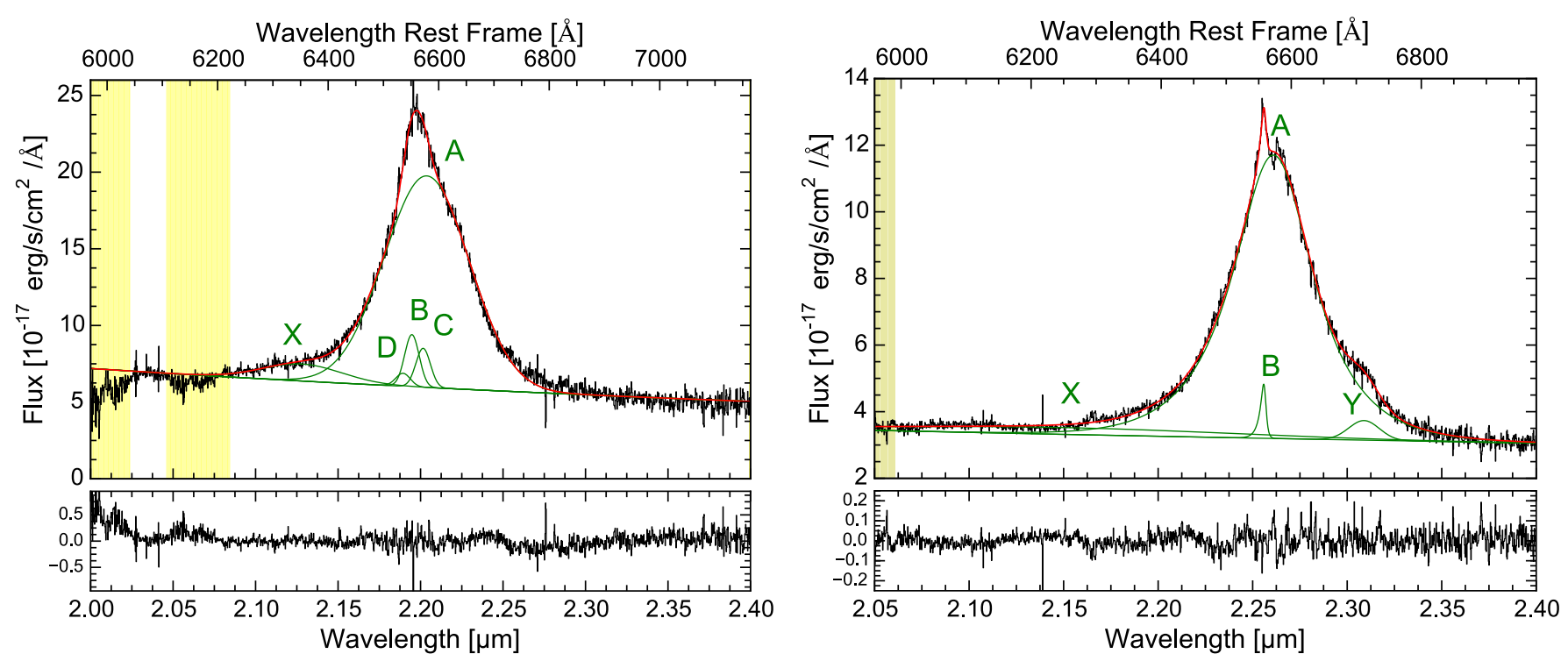

Fig. 5. $K$-band SINFONI spectra of LBQS0109 (left panel) and HB8903 (right panel) in the spectral region of H $\alpha$ integrated in a region of $0.25^{\prime \prime} \times 0.25^{\prime \prime}(2 \times 2$ pixel $)$ around the QSO. Upper panels: observed spectra shown with a black line. The different components in the fit for each line ( $\mathrm{H} \alpha,[\mathrm{NII}]$, and continuum) are shown in green, and the red line shows the total fit. The shaded yellow regions indicate the zone affected by strong sky line residuals, which are excluded from the fit. Bottom panels: fit residuals, obtained as a difference between observed and model spectra.

component is consistent with the typical line widths observed in star-forming galaxies at $z \sim 2.5$ (Förster Schreiber et al. 2009).

\subsection{SED fitting}

Figure 7 shows the rest-frame spectral energy distributions (SEDs) of the two QSOs obtained by combining Sloan Sky Digital Survey (SDSS; when available), Two Micron All Sky Survey (2MASS) and Wide-field Infrared Survey Explorer (WISE) photometry. We did not include photometric points at rest-frame wavelength $<1215.67 \AA$ since such emission is affected by intergalactic medium absorption. We took advantage of 2MASS data in the $J(1.24 \mu \mathrm{m}), H(1.66 \mu \mathrm{m})$ and $K(2.16 \mu \mathrm{m})$ bands. The photometric points were extracted from All Sky Point Source Catalogue. We used the data observed with the infrared telescope WISE in the $W 1(3.4 \mu \mathrm{m}), W 2(4.6 \mu \mathrm{m})$, $W 3(12 \mu \mathrm{m})$, and $W 4(22 \mu \mathrm{m})$ bands. The continuum fluxes for both targets in all four bands can be found in the WISE All-Sky Source Catalogue. For LBQS0109, we extracted the continuum flux from four out of five SDSS colour bands $(g, r, i$, and $z)$, selecting the parameter psfMag that measured the total flux of the object by fitting a PSF model in the position of the target.

The SED fitting decomposition, shown as a red solid line in Fig. 7, was performed by using the SED fitting code by Balmaverde et al. (2016), who assumed that the best-fit SED is the combination of different components: accretion disk emission, unabsorbed (old) stellar population, emission of dust heated by the AGN (black body at $\sim 1500 \mathrm{~K}$ and torus template), and by a starburst emission (starburst template). We refer to Balmaverde et al. (2016) for a detailed description of the adopted templates and fitting procedure. The lack of photometric points in the rest frame $8-1000 \mu \mathrm{m}$ does not allow us to constrain the presence of a possible starburst component. However, we note that such a component is not required by the SED fitting performed with the current data.

The colour-filled areas indicate the uncertainties on the best fit, which were estimated through a Monte Carlo procedure, see
Balmaverde et al. (2016) for more details. Clearly, the lack of photometric points beyond $10 \mu \mathrm{m}$ rest-frame does not allow us to constrain the emission from the AGN torus in either source. The luminosity at $1-3 \mu \mathrm{m}$ can be explained with the emission from an unobscured population of old stars in the host galaxy (Meidt et al. 2012). From the SED fitting, we derive a stellar mass (hereafter $M_{\star}$ ) of $1.5 \times 10^{12} M_{\odot}$ for LBQS0109 and of $0.6 \times 10^{12} M_{\odot}$ for HB8903, the latter with a large uncertainty. However, since the old star components contribute less than $25 \%$ to the total luminosity in the range between $1 \mu \mathrm{m}$ and $3 \mu \mathrm{m}$, we consider the inferred $M_{\star}$ as upper limits.

\section{Discussion}

In the following we discuss the interpretations of the two narrow components, [OIII $]_{\text {nar }}$ and $\mathrm{H} \alpha_{\text {nar }}$, detected in the two QSOs. The surface brightness of $\mathrm{H} \alpha$ is similar to that observed in previous works (Cano-Díaz et al. 2012; Cresci et al. 2015a) and suggests two possible scenarios.

\subsection{Negative-feedback scenario}

Figure 8 shows the flux maps of the $\mathrm{H} \alpha_{\text {nar }}$ component obtained by collapsing the residual data cubes of LBQS0109 and for HB8903 over the wavelength bins where $\mathrm{H} \alpha_{\text {nar }}$ is detected. The $\mathrm{H} \alpha_{\text {nar }}$ emission is extended to $\sim 1^{\prime \prime}(\sim 8 \mathrm{kpc})$ from the AGN in both sources. Moreover, the $\mathrm{H} \alpha_{\text {nar }}$ surface brightness is asymmetrically distributed around the nucleus, similarly to [OIII] $\lambda 5007$. The $\mathrm{H} \alpha$ nar emission in LBQS0109 is primarily distributed toward the S-E, while that of HB8903 is concentrated in three different clumps located N-W and S-W of the AGN position. The $\mathrm{H} \alpha_{\text {nar }}$ surface brightness maps are clearly similar to those obtained for $[\mathrm{OIII}]_{\text {nar }}$ shown in Fig. 4.

The white contours in Fig. 8 show the most blue-shifted [OIII] $\lambda 5007$ projected velocities $\left(-200,-180\right.$, and $-150 \mathrm{~km} \mathrm{~s}^{-1}$ for LBQS0109, and $-150,-100$, and $-50 \mathrm{~km} \mathrm{~s}^{-1}$ for HB8903) and therefore correspond to the regions where the fastest 

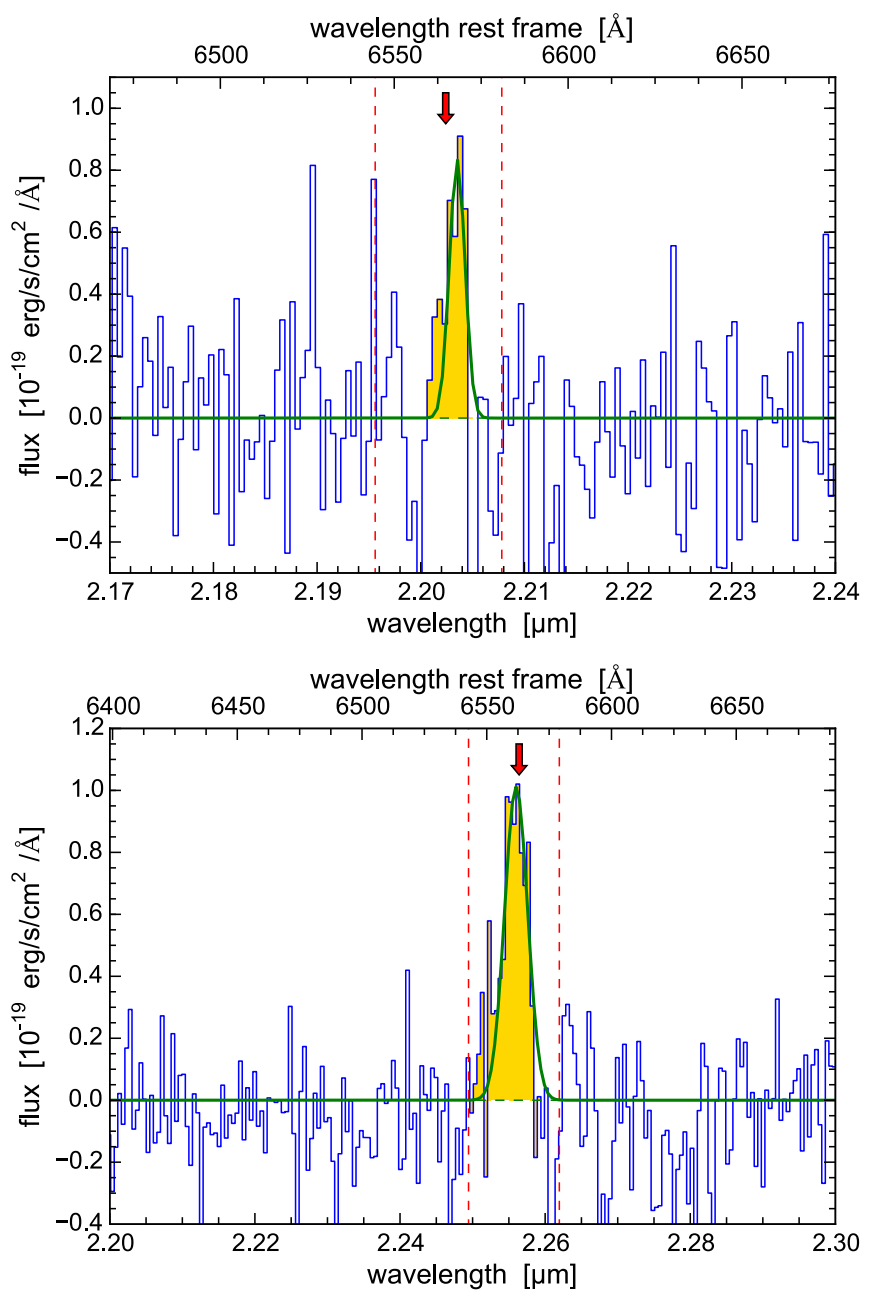

Fig. 6. Narrow $\mathrm{H} \alpha$ emission from the residual $K$-band fitting, extracted from a ring-shaped region $\left(0.5^{\prime \prime}<r<0.8^{\prime \prime}\right)$ of LBQS0109 (top) and HB8903 (bottom). The red arrows indicate the expected position at the redshift of the $[\mathrm{OIII}]_{\mathrm{nar}}$ component. The dashed red line indicates the expected positions for doublet [NII]: the lines are not detected, confirming that star formation is the excitation mechanism for the narrow $\mathrm{H} \alpha$ line (see text).

[OIII] $\lambda 5007$ outflows are located: these appear spatially anticorrelated with the $\mathrm{H} \alpha_{\text {nar }}$ emission. We interpret these observations as evidence for negative feedback in action because they indicate that star formation is quenched in the regions where AGNdriven outflows interact with the host galaxy. The present data do not allow us to distinguish between the possibilities that in the regions encompassed by the fast outflows, star formation is absent due to missing molecular gas or because molecular gas has been heated or made more turbulent. Our planned ALMA observations of the $\mathrm{CO}(3-2)$ line at a resolution similar to the SINFONI observations will address this question.

We now try to assess whether these AGN-driven outflows significantly quench the SFR of the whole galaxy, beyond the regions shown in Fig. 8.

To establish whether the total star formation in the QSO host galaxies is significantly quenched, the location of these sources could be studied in the $S F R-M_{\star}$ plane and be compared with the main sequence (MS) of star formation at $z \sim 2$ : a significant quenching of star formation in the hosts of the two quasars could be revealed by these being significantly below the MS.
We estimated the SFR in the quasar host galaxies from the $\mathrm{H} \alpha_{\text {nar }}$ emission, assuming a Chabrier initial mass function (Chabrier 2003). It was not possible to correct for reddening because we were unable to obtain a reliable measurement or a significant upper limit on the narrow $\mathrm{H} \beta$ emission. Therefore our SFR estimates are very likely lower limits. By using the Kennicutt \& Evans (2012) relation $\log \left(S F R / M_{\odot} \mathrm{yr}^{-1}\right)=$ $\log \left(L_{\mathrm{H} \alpha} / \mathrm{erg} \mathrm{s}^{-1}\right)-41.27$, we derived a total SFR from $\mathrm{H} \alpha_{\text {nar }}$ of $S F R \sim 50 M_{\odot} \mathrm{yr}^{-1}$ for LBQS0109 and SFR $\sim 90 M_{\odot} \mathrm{yr}^{-1}$ for HB8903, which is consistent with the wide ranges of SFR observed in star-forming and QSO host galaxies at similar redshifts (e.g. Lutz et al. 2008; Cano-Díaz et al. 2012; Cresci et al. 2015a; Netzer et al. 2016). The detected $\mathrm{H} \alpha$ emission traces the instantaneous SFR, while most works that have tried to estimate the MS for SFR-stellar mass relation at high red-shift used farinfrared (FIR; $\lambda=8-1000 \mu \mathrm{m}$ ) or a combination of FIR and ultraviolet (UV) observations and therefore provided the timeintegrated SFR, which can be considerably higher than the SFR obtained from $\mathrm{H} \alpha$.

The SED fitting presented in Sect. 3.3 does not allow us to reliably estimate stellar masses but only upper limits, which are $1.5 \times 10^{12} M_{\odot}$ and $6.0 \times 10^{11} M_{\odot}$ for LBQS0109 and HB8903, respectively.

From combining the uncertainty on the SFR with the upper limits on the stellar masses, we conclude that it is not possible to constrain the location of the quasar host galaxy sources on the SFR- $M_{\star}$ plane. We only note that an MS star-forming galaxy with $M_{\star}=5 \times 10^{11} M_{\odot}$ at $z \sim 2$ has an $S F R_{\mathrm{MS}}$ of $\sim 220 M_{\odot} \mathrm{yr}^{-1}$ (Whitaker et al. 2012). Since this is a factor $\sim 2-4$ higher than the value we inferred from $\mathrm{H} \alpha$, the two host galaxies are probably below the MS and, hence, may be quenched. However, the lack of FIR observations do not allow us to confirm this point.

Considering the uncertainties on SFR estimates, the upper limits on stellar masses, and the intrinsic scatter of the MS of star formation, we conclude that it is not possible to establish whether the AGN-driven outflows significantly quench star formation in the whole galaxy.

\subsection{Positive-feedback interpretation}

Our observations vaguely resemble the observations at a $z=$ 1.5 source (XID2028) by Cresci et al. (2015a), where the ionised outflow was found to anti-correlate with the $\mathrm{H} \alpha$ emission and the rest-frame $U$-band surface brightness, which map the current star formation in the host galaxy. The two emissions lie in both edges of the outflow cone. These observations were interpreted in terms of both negative and positive feedback, where the fast wind sweeps up the interstellar gas of the host along the core of the outflow (i.e. negative feedback) and the ionised outflow simultaneously triggers star formation by compressing the gas clouds (i.e. positive feedback).

In our sources and in that of Cano-Díaz et al. (2012), star formation is distributed along both edges of the outflow cone (Fig. 8) as shown in the schematic view in Fig. 5 of Cresci et al. (2015a). Therefore, it is also possible to interpret our results in terms of positive feedback, which has been invoked by recent models to explain the correlation between AGN and star formation activity in the host galaxy (Silverman et al. 2009; Imanishi et al. 2011; Mullaney et al. 2012; Zinn et al. 2013; Zubovas et al. 2013). This scenario may be confirmed by the fact that $\mathrm{H} \alpha$ emission is mainly produced by massive stars with lifetimes of $<10$ Myr. This time is as long as typical AGN activity, suggesting that the star formation activity is connected with AGNdriven outflows. However, we cannot exclude that the current 

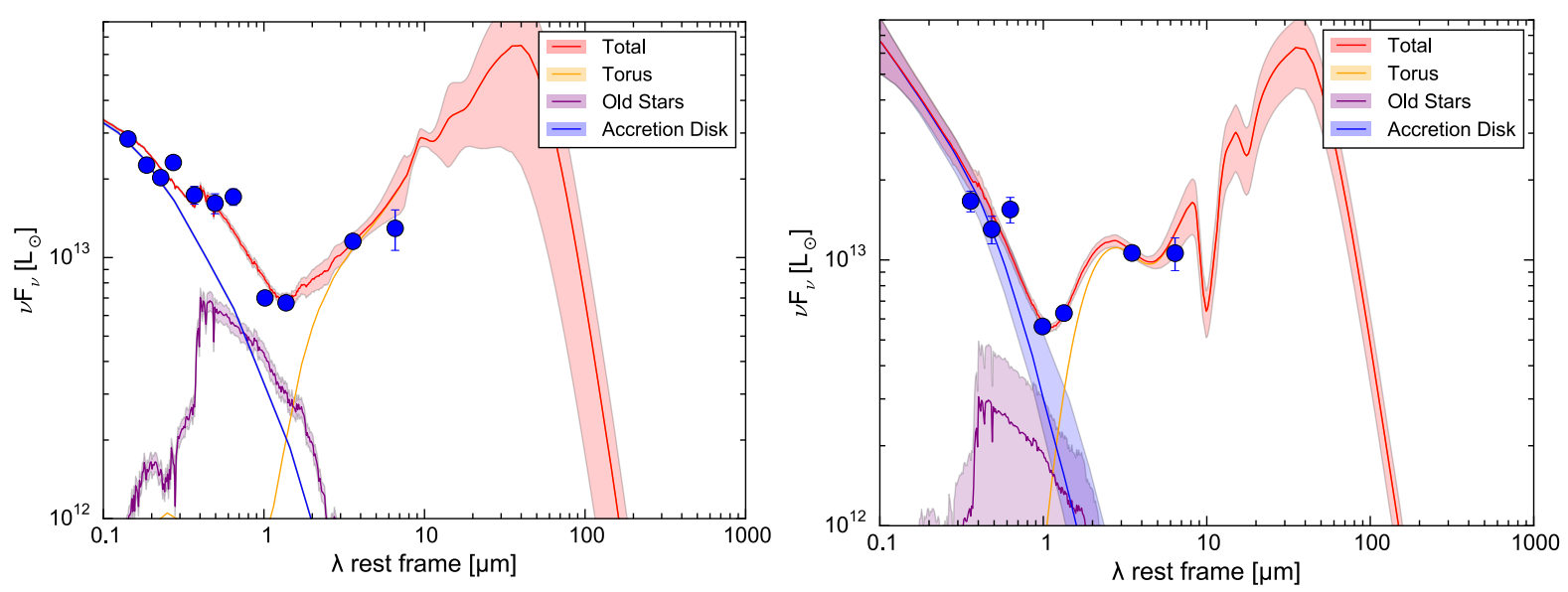

Fig. 7. SED fitting of LBQS0109 (left) and HB8903 (right). The blue points show the QSO emission fluxes from SDSS, 2MASS, and WISE photometry. The total best-fit models are plotted in red, while accretion disk, old star, and torus components are plotted in blue, purple, and orange, respectively. The filled areas denote the uncertainties estimated with a Monte Carlo procedure, see Balmaverde et al. (2016) for more details.
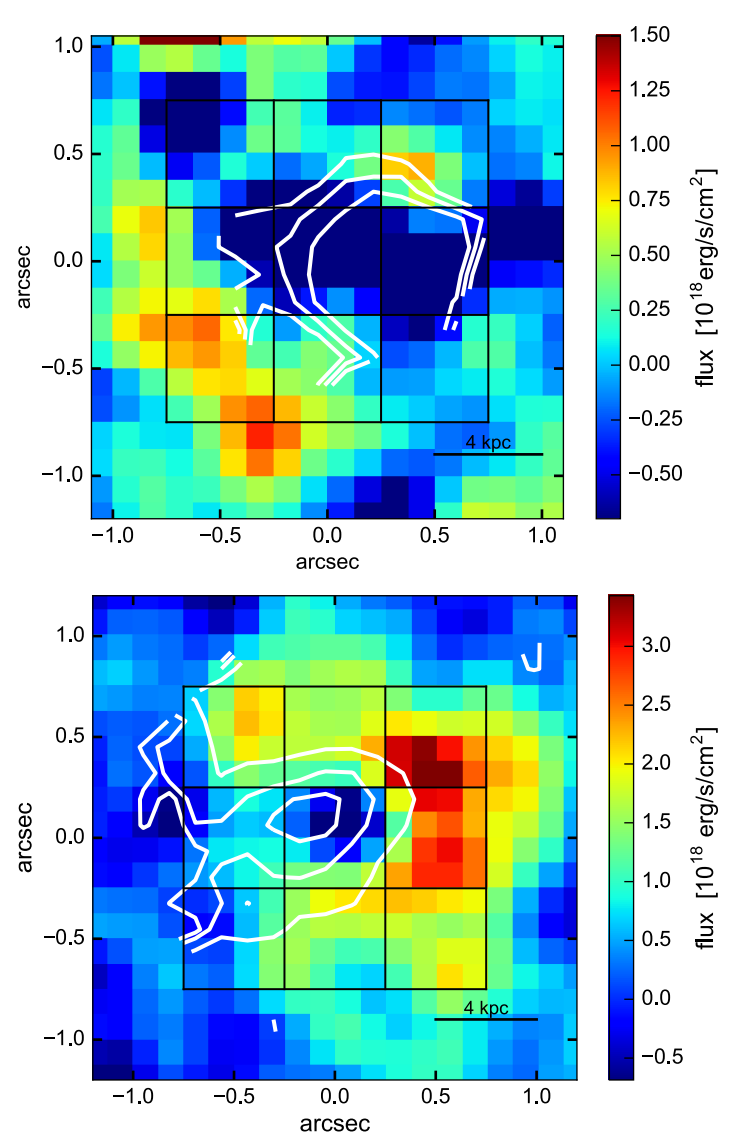

Fig. 8. Maps of the narrow component of $\mathrm{H} \alpha$ for LBQS0109 (top) and HB8903 (bottom); the white contours trace the [OIII] $\lambda 5007$ velocity blue-shift shown in Fig. 3. The contours represent the velocity -300 , -275 , and $-250 \mathrm{~km} \mathrm{~s}^{-1}$ for LBQS0109, and $-150,-100$, and $-50 \mathrm{~km} \mathrm{~s}^{-1}$ for HB8903. Star formation traced by $\mathrm{H} \alpha_{\text {nar }}$ is heavily suppressed in the S-W and S-E for region for LBQS0109 and HB8903, respectively, where the outflow traced by [OIII] $\lambda 5007$ is fastest. Black squares indicate the nine regions where we extracted the corresponding $H$-band spectra shown in the right panels of Fig. 1.

SFR in the host galaxies is a residual of SF activity occurring before the negative feedback phase. SFRs in the host galaxies of bright QSOs can be as high as $1000 M_{\odot} \mathrm{yr}^{-1}$, hence an
$S F R<100 M_{\odot} \mathrm{yr}^{-1}$ might imply a significant reduction compared to the past. In this case, the existing star formation regions would not be induced by positive feedback, but would be regions of star formation that is not perturbed by AGN-driven outflows.

\section{Conclusions}

The two QSOs LBQS0109 and HB8903, part of a sample of six presented in a previous paper (Carniani et al. 2015), clearly show evidence of [OIII] $\lambda 5007$ emission from the host galaxy in their SINFONI $H$-band spectra. The [OIII] $\lambda 5007$ emission is characterised by a broad blue-shifted component, tracing a powerful outflow (Paper I), and by a narrow red-shifted component $\left(F W H M<500 \mathrm{~km} \mathrm{~s}^{-1}\right)$ that is most likely associated with star-forming regions in the host galaxies. Our new SINFONI $K$-band observations confirm this hypothesis. After subtracting pixel-by-pixel all broad $\left(F W H M>500 \mathrm{~km} \mathrm{~s}^{-1}\right) \mathrm{H} \alpha$ and [NII] components from $K$-band spectra, we detected faint narrow $\left(F W H M \sim 250-500 \mathrm{~km} \mathrm{~s}^{-1}\right) \mathrm{H} \alpha_{\text {nar }}$ emission at the same redshift of $[\mathrm{OIII}]_{\text {nar }}$ that is co-spatially distributed with this line in both QSOs.

The emitted $\mathrm{H} \alpha_{\text {nar }}$ flux is not symmetrically distributed around the location of the QSOs, but is extended towards the SE and W for LBQS0109 and HB8903, respectively. Line widths and surface brightness distributions of these components both suggest that they are powered by SF in the host galaxies. The inferred upper limit on $\log ([\mathrm{NII}] / \mathrm{H} \alpha)(-0.85$ for LBQS0109 and -1.32 for HB8903) is consistent with the typical value observed in star-forming regions, according to the standard BPT diagrams. For LBQS0109, we cannot exclude the possibility that the $[\mathrm{OIII}]_{\text {nar }}$ and $\mathrm{H} \alpha_{\text {nar }}$ emissions are associated with AGN emission if we assume a metal-poor AGN at $z \sim 2.5$ (panels $2 \mathrm{~d}$ and $4 \mathrm{~d}$ of Fig. 5 of Kewley et al. 2013).

Summarising, with this work we doubled the sample of QSOs (from two to four) where the effects of AGN-driven feedback on star formation are clearly visible. We note that in all these cases the SFRs inferred from the narrow $\mathrm{H} \alpha$ emission is significant ( $\left.\sim 50-90 M_{\odot} \mathrm{yr}^{-1}\right)$ and, as a result of the unknown dust obscuration on the narrow $\mathrm{H} \alpha$, they are very likely below the total SFR in the host galaxies.

Although the statistics is still limited, the combined observations reinforce the idea that quasar outflows do affect only a (small) part of the host galaxy; therefore either AGN feedback 
does not completely quench star formation, or several AGN episodes are needed to accomplish this. A larger sample with similar $L_{\mathrm{AGN}}$ and $z$ will allow us to reach more reliable results. In particular, a comparison of the SFR between sources that show evidence for fast ionised outflows and those that do not will resolve the negative-feedback puzzle.

On the other hand, our results may be consistent with the scenario proposed by Cresci et al. (2015a), in which outflows remove gas along the direction of motion and, simultaneously, compress the gas at the edges of the outflow cone, triggering star formation. Thus AGN-driven outflows might both quench and induce star formation in host galaxies.

Acknowledgements. We acknowledge financial support from INAF and the Italian Ministry of University and Research under the contracts PRIN-INAF2011 ("Black Hole growth and AGN feedback through cosmic time") and PRIN MIUR 2010-2011 ("The dark Universe and the cosmic evolution of baryons"). M.B. acknowledges support from the FP7 Career Integration Grant "eEASy" (CIG 321913). R.S. acknowledges support from the European Research Council under the European Union (FP/2007-2013)/ERC Grant Agreement n. 306476. E.P. acknowledges financial support from INAF under the contract PRIN-INAF-2012. C.C. gratefully acknowledges support from the Swiss National Science Foundation Professorship grant PP00P2_138979/1 (ETH Zurich). We thank the anonymous referee for comments and suggestions that improved the paper.

\section{References}

Aalto, S., Garcia-Burillo, S., Muller, S., et al. 2015, A\&A, 574, A85 Baldry, I. K., Glazebrook, K., Brinkmann, J., et al. 2004, ApJ, 600, 681 Baldwin, A., Phillips, M. M., \& Terlevich, R. 1981, PASP, 93, 817 Balmaverde, B., Marconi, A., Brusa, M., et al. 2016, A\&A, 585, A148 Brusa, M., Bongiorno, A., Cresci, G., et al. 2015, MNRAS, 446, 2394 Cano-Díaz, M., Maiolino, R., Marconi, A., et al. 2012, A\&A, 537, L8 Carniani, S., Marconi, A., Maiolino, R., et al. 2015, A\&A, 580, A102 (Paper I) Chabrier, G. 2003, PASP, 115, 763

Cicone, C., Maiolino, R., Gallerani, S., et al. 2015, A\&A, 574, A14 Cicone, C., Feruglio, C., Maiolino, R., et al. 2012, A\&A, 543, A99 Cicone, C., Maiolino, R., Sturm, E., et al. 2014, A\&A, 562, A21 Costa, T., Sijacki, D., \& Haehnelt, M. G. 2014, MNRAS, 444, 2355 Costa, T., Sijacki, D., \& Haehnelt, M. G. 2015, MNRAS, 448, L30 Cresci, G., Mainieri, V., Brusa, M., et al. 2015a, ApJ, 799, 82 Cresci, G., Marconi, A., Zibetti, S., et al. 2015b, A\&A, 582, A63 Davies, R. I. 2007, MNRAS, 375, 1099

Di Matteo, T., Springel, V., \& Hernquist, L. 2005, Nature, 433, 604 Fabian, A. C. 2012, ARA\&A, 50, 455

Faucher-Giguère, C.-A., \& Quataert, E. 2012, MNRAS, 425, 605 Ferrarese, L., \& Merritt, D. 2000, ApJ, 539, L9
Feruglio, C., Fiore, F., Maiolino, R., et al. 2013a, A\&A, 549, A51 Feruglio, C., Fiore, F., Piconcelli, E., et al. 2013b, A\&A, 558, A87 Feruglio, C., Fiore, F., Carniani, S., et al. 2015, A\&A, 583, A99 Förster Schreiber, N. M., Genzel, R., Bouché, N., et al. 2009, ApJ, 706, 1364 Gebhardt, K., Bender, R., Bower, G., et al. 2000, ApJ, 539, L13

Granato, G. L., De Zotti, G., Silva, L., Bressan, A., \& Danese, L. 2004, ApJ, 600,580

Harrison, C. M., Alexander, D. M., Swinbank, A. M., et al. 2012, MNRAS, 426, 1073

Harrison, C. M., Alexander, D. M., Mullaney, J. R., \& Swinbank, A. M. 2014, MNRAS, 441, 3306

Harrison, C. M., Alexander, D. M., Mullaney, J. R., et al. 2016, MNRAS, 456, 1195

Hopkins, P. F., Somerville, R. S., Hernquist, L., et al. 2006, ApJ, 652, 864 Husemann, B., Jahnke, K., Sánchez, S. F., et al. 2014, MNRAS, 443, 755 Imanishi, M., Ichikawa, K., Takeuchi, T., et al. 2011, PASJ, 63, 447

Kauffmann, G., Heckman, T. M., Tremonti, C., et al. 2003, MNRAS, 346, 1055 Kennicutt, R. C., \& Evans, N. J. 2012, ARA\&A, 50, 531

Kewley, L. J., Dopita, M. A., Leitherer, C., et al. 2013, ApJ, 774, 100

King, A. R. 2010, MNRAS, 402, 1516

King, A., \& Pounds, K. 2015, ARA\&A, 53, 115

Kormendy, J., \& Ho, L. C. 2013, ARA\&A, 51, 511

Lutz, D., Sturm, E., Tacconi, L. J., et al. 2008, ApJ, 684, 853

Magorrian, J., Tremaine, S., Richstone, D., et al. 1998, AJ, 115, 2285

Maiolino, R., Gallerani, S., Neri, R., et al. 2012, MNRAS, 425, L66

Marconi, A., \& Hunt, L. K. 2003, ApJ, 589, L21

Marconi, A., Risaliti, G., Gilli, R., et al. 2004, MNRAS, 351, 169

Meidt, S. E., Schinnerer, E., Knapen, J. H., et al. 2012, ApJ, 744, 17

Menci, N., Fiore, F., Puccetti, S., \& Cavaliere, A. 2008, ApJ, 686, 219

Mullaney, J. R., Daddi, E., Béthermin, M., et al. 2012, ApJ, 753, L30

Nayakshin, S. 2014, MNRAS, 437, 2404

Netzer, H., Shemmer, O., Maiolino, R., et al. 2004, ApJ, 614, 558

Netzer, H., Lani, C., Nordon, R., et al. 2016, ApJ, 819, 123

Pérez-González, P. G., Trujillo, I., Barro, G., et al. 2008, ApJ, 687, 50

Perna, M., Brusa, M., Cresci, G., et al. 2015a, A\&A, 574, A82

Perna, M., Brusa, M., Salvato, M., et al. 2015b, A\&A, 583, A72

Planck Collaboration XVI. 2014, A\&A, 571, A16

Reines, A. E., \& Volonteri, M. 2015, ApJ, 813, 82

Roos, O., Juneau, S., Bournaud, F., \& Gabor, J. M. 2015, ApJ, 800, 19

Rupke, D. S. N., \& Veilleux, S. 2013, ApJ, 775, L15

Shemmer, O., Netzer, H., Maiolino, R., et al. 2004, ApJ, 614, 547

Silverman, J. D., Kovač, K., Knobel, C., et al. 2009, ApJ, 695, 171

van Dokkum, P. G. 2001, PASP, 113, 1420

Whitaker, K. E., van Dokkum, P. G., Brammer, G., \& Franx, M. 2012, ApJ, 754, L29

Young, J. E., Eracleous, M., Shemmer, O., et al. 2014, MNRAS, 438, 217

Zinn, P.-C., Middelberg, E., Norris, R. P., \& Dettmar, R.-J. 2013, ApJ, 774, 66

Zubovas, K., \& King, A. 2012, ApJ, 745, L34

Zubovas, K., \& King, A. R. 2014, MNRAS, 439, 400

Zubovas, K., Nayakshin, S., King, A., \& Wilkinson, M. 2013, MNRAS, 433, 3079 